\title{
To Resolve the Problems of Judgment Aggregation from the Perspective of Logic
}

\author{
Li Dai \\ School of Literature and Chinese as a Foreign Language, Mianyang Normal University, Province Sichuan, \\ China
}

Jihua Zhang

Department of Science and technology, Mianyang Normal University, Province Sichuan, China

\begin{abstract}
It is a research hotspot to study the social choice theory from the perspective of logic in recent years. The judgment aggregation model based on logic supports a new way for the research of social choice theory. We attempt to analyze judgment aggregation dilemmas based on the judgment aggregation model, explore different aggregation problem solutions.
\end{abstract}

KEYWORD: Logic; Judgment aggregation; Escape routs

\section{INTRODUCTION}

Since the classic works Social Choice and Individual values of the American economist Professor Kenneth Arrow emerged, social choice theory research becomes more and more hot. It is a research hotspot to study the social choice theory from the perspective of logic in recent years. The main question social choice theory concerns about is that how does a group aggregate the individual opinions together to make a group decision. The significant decision-making problems are generally resolved by group decision in real life.

Preference aggregation and judging aggregation are two basic studying frameworks of social choice theory. Close relation exists between them. In Social Choice and Individual values Arrow studied issues of preference aggregation, namely whether we can get a group decision based on the known personal preferences and make the process meet certain natural conditions. The research of social choice theory has mainly focused on the preference aggregation (or individual utility aggregation) for nearly half a century because of the widely applying of preference aggregation problem and the unique charm of the axiomatic method for studying social choice theory.

But on many occasions, we need not only aggregate individual preferences, but also aggregate individual judgments. For example, a discussion group composed of three experts wants to get a group judgment (that is "yes" or "no") for the following three propositions logically interrelated:

p: Carbon emissions are above a given threshold;

$\mathrm{p} \rightarrow \mathrm{q}$ : If Carbon emissions are above a given threshold, there will be a critical temperature increase;

q: There will be a critical temperature increase.

Suppose now that the three experts disagree the case, as shown in Table 1.

Table 1 A problem of inconsistent majority judgment

\begin{tabular}{|c|c|c|c|}
\hline & $\mathrm{p}$ & $\mathrm{p} \rightarrow \mathrm{q}$ & $\mathrm{q}$ \\
\hline expert 1 & false & true & false \\
\hline expert 2 & true & false & false \\
\hline expert 3 & true & true & true \\
\hline majority & true & true & false \\
\hline
\end{tabular}

The first thinks that $\mathrm{p} \rightarrow \mathrm{q}$ is true, $\mathrm{p}$ and $\mathrm{q}$ are both false. The second thinks that $p$ is true, $p \rightarrow q$ and $q$ are both false. The third thinks that $p$ and $p \rightarrow q$ are both true, $\mathrm{q}$ is true as well. Then each expert holds consistent judgments on the three propositions, yet there are majorities for $\mathrm{p}$, for $\mathrm{p} \rightarrow \mathrm{q}$ and for $\neg \mathrm{q}$, a logically inconsistent set of propositions. The problem is very general.

\section{THE JUDGMENT AGGREGATION MODEL BASED ON LOGIC}

In order to ask whether other aggregation rules may be immune to the problem of inconsistent majority judgment, it is necessary to introduce a more general model.

Let $N=\{1,2, \ldots, \mathrm{n}\}$ be a finite set of individuals. Let $\mathrm{L}$ be a logic with language $\mathscr{L}(\mathrm{L})$ which has the usual semantics. Relevant concepts are as follows:

(1) agenda: An agenda over L is a non-empty set $A \subseteq \mathcal{L}(\mathrm{L})$, where for every formula $\Phi$ that does not start with a negation, $\Phi \in A$ if and only if 
$\neg \Phi \in A$. We sometimes call a member of $A$ an agenda item.

(2) consistent: $A$ set $A \subseteq \mathcal{L}(\mathrm{L})$ is consistent unless $\mathrm{A} \vdash_{\mathrm{L}} \Phi \wedge \neg \Phi$ for some $\Phi \in \mathscr{L}(\mathrm{L})$.

(3) complete: $A$ set $A \subseteq \mathcal{L}(L)$ is complete if either $\Phi \in \mathrm{A}$ or $\neg \Phi \in \mathrm{A}$ for every $\Phi \in A$ which does not start with negation.

(4) judgment set: A judgment set is a complete and consistent subset $A \subseteq A$.

(5) judgment profile: An judgment profile is an ntuple $\left(A_{1}, \ldots, A_{n}\right)$, where $A_{i}(1 \leq i \leq n)$ is the individual judgment set of agent i. $\mathrm{J}(A, \mathrm{~L})$ denotes the set of all individual (complete and L-consistent) judgment sets over $A$. When $\gamma \in \mathrm{J}(A, \mathrm{~L})^{\mathrm{n}}$, we use $\gamma_{\mathrm{i}}$ to denote the ith element of $\gamma$, i.e., agent i's individual judgment set in judgment profile $\gamma$.

(6) judgment aggregation rule: A judgment aggregation rule is a function $f$ : $J(A, \mathrm{~L})^{n} \rightarrow J(A, \mathrm{~L})$.

The judgment aggregation rule should usually meet the following conditions:

(1) Independence: The group's judgment on any agenda item in an agenda only depends on individuals' judgment on this agenda item and has nothing to do with other factors.

(2) Unanimity: If all individuals agree with certain agenda item in the agenda, then the group holds the same view.

(3) Non-dictatorship: We should not ignore others' different judgment and make a fixed individual's judgment always be the group's.

Discursive Dilemma: If there are at least three subjects need to make a judgment on at least two different formulas $\mathrm{p}, \mathrm{q}$, and $\mathrm{p} \rightarrow \mathrm{q}$, then we may find out an inconsistent group judgment if we adopt majority vote aggregation rule.

Similar to Arrow's Theorem and many other impossibility theorems, there are also several impossibility theorems in judgment aggregation. Because of the advantages of judgment aggregation which preference aggregation does not have, the social choice theory researchers gradually focus on judgment aggregation. Judgment aggregation model based on logic explores a new road for researchers on social choice theory and also provides new ideas to solve problems in social choice theory.

\section{ESCAPE ROUTES FROM THE PROBLEMS OF JUDGMENT AGGREGATION}

The impossibility theorems in judgment aggregation not only prove that there is no perfect rule for aggregation, but also make us realize that we should relax these requirements to find an aggregation rule which can be accepted.

\subsection{Restricting the domain}

The impossibility theorems in judgment aggregation take the aggregation rule to be defined on the domain of all judgment profiles which consist of any consistent and complete individual judgment sets and place no restriction. Dietrich and List propose that whether there is admissible restriction of domain to make sure that majority judgment of agenda items is consistent.[10] Some restrictions, which are discussed, are similar to some classic domain restrictions in preference aggregation(such as single-peakedness). The method of unidimensionally aligned is based on the idea to restrict the domain. A judgment profile is unidimensionally aligned if the individuals in $N$ can be ordered from left to right such that the individuals who agree $\mathrm{p}$ for every proposition $\mathrm{p}$ in the agenda. Consider the following example, the agenda contains the following propositions and their negations:

p: Carbon emissions are above a given threshold;

$\mathrm{p} \rightarrow \mathrm{q}$ : If Carbon emissions are above a given threshold, there will be a critical temperature increase;

q: There will be a critical temperature increase.

As is shown in Table 2: the result is unidimensionally aligned. Any proposition in the agenda is agreed by majority if and only if it is agreed by the median individual on the given leftright order. Here the majority judgments(group judgments) are those of individual 3. For any given judgment set of all individuals consistent, thus the majority judgment set is consistent.

The method of unidimensionally aligned is strong domain-restriction condition in judgment aggregation. Only if the number of individuals is odd number, this method may be effective and must be invalid when the number of individuals is even number.

Table 2 A unidimensionally aligned judgment profile

\begin{tabular}{|c|c|c|c|c|c|}
\hline & Ind.1 & Ind.2 & Ind.3 & Ind.4 & Ind.5 \\
\hline $\mathrm{p}$ & $\mathrm{A}$ & $\mathrm{A}$ & $\mathrm{O}$ & $\mathrm{O}$ & $\mathrm{O}$ \\
\hline $\mathrm{p} \rightarrow \mathrm{q}$ & $\mathrm{O}$ & $\mathrm{O}$ & $\mathrm{A}$ & $\mathrm{A}$ & $\mathrm{A}$ \\
\hline $\mathrm{q}$ & $\mathrm{O}$ & $\mathrm{O}$ & $\mathrm{O}$ & $\mathrm{A}$ & $\mathrm{A}$ \\
\hline
\end{tabular}

(A represents "agree" and O represents "oppose")

\subsection{Relaxing of restriction to the outputs}

Instead of restricting the domain of inputs to the aggregation rule, we can also relax the restricting to the outputs. The impossibility theorems in judgment aggregation require the aggregation rules to generate a complete, not just consistent group judgment set for every profile of individual judgment sets. But there are many contexts in which completeness seems too much to ask of group judgments. Two common judgment aggregation rules violating 
completeness are unanimity rules and supermajority rules.[9]

The unanimity rules means that the group agrees with a certain agenda item if and only if every individual agrees with the agenda item. The supermajority rules means that the group agrees with a certain agenda item if and only if a sufficiently large supermajority, such as $2 / 3,3 / 4$, or more individuals agree with the agenda item. An important difference between the two rules lies in the rationality conditions on group judgments they secure. When a judgment set is consistent and complete, it is also deductively closed. Accordingly, when we relax completeness, we must choose whether or not to preserve deductive closure.

\subsection{Relaxing independence}

Weakening the conditions of independence is another way to resolve problems of judgment aggregation. In preference aggregation, independence is usually defended by appealing to strategy-proofness. But it seems less natural to require the court's judgments on the defendant's liability to be independent of the individuals' judgments on whether he did it or whether he was obliged not to. Two common judgment aggregation rules relaxing independence are premise-based rules and conclusion-based rules.

The basic idea of premise-based rules is to select a subset of the agenda as a set of premises and to aggregation these premises through a certain judgment rule of independence, but to allow the group judgments on the other propositions according to the group's judgment on these premises. Consider the example of experts discussion group in general instruction. $\{\mathrm{p}, \neg \mathrm{p}, \mathrm{p} \rightarrow \mathrm{q}, \neg(\mathrm{p} \rightarrow \mathrm{q})\}$ can be taken as premises. For these premises, we can aggregate the group judgment set $\{\mathrm{p}, \mathrm{p} \rightarrow \mathrm{q}\}$ according to majority rule. From the group judgment set $\{p, p \rightarrow q\}$, we can obtain that the group agrees with $\mathrm{q}$. Conclusionbased rules, by contrast, apply a propositionwise independent aggregation rule directly on the conclusions.

\section{CONCLUSION}

How to aggregate individual rationality into group rationality is the main problem faced by the cognitive study of social rationality, it is also one of the hot interdisciplinary questions among economics, philosophy, politics, law and computer science etc. The famous American economist Professor Kenneth Arrow used the axiomatic method depicting individual rationality and group rationality, and proved the impossibility of aggregating the rational individual preference into the rational group preference.[4] It becomes an important problem in many studying areas that how to understand the impossibility and to dissolve it for finding reasonable group rationality aggregating methods, judgment aggregation provided a new idea for the breakthrough of the problem. The impossibility conclusions in the areas of preference aggregation and judgment aggregation revealed the impossibility to aggregate individual rationality into group rationality under some reasonable conditions. In the paper, we study the collective rationality aggregation problems based on the judgment aggregation model, so as to explore feasible escape routes for it. This research would lay theoretical foundation for making group decision schemes, thus further promote the formal study of social collective cognition.

\section{ACKNOWLEDGEMENT}

This paper is one of the phased objectives of scientific researcher fund project "Study on Group Rational Conditions Based on Logic of Judgment Aggregation" (GN: QD2012B03) of Mianyang Normal University and scientific project "Study on Group Ration Aggregation from the Perspective of Modern Loic" (GN:13SB0159) of Sichuan education department.

\section{REFERENCES}

[1] ̊̊gotnes, T., Hoek W. \& Wooldridge, M. 2006. Towards a logic of social welfare. The 7th Conference on Logic and the Foundations of Game and Decision Theory (LOFT 06).

[2] Ågotnes, T., Hoek, W. \& Wooldridge, M. 2011. On the logic of preference and judgment aggregation. Autonomous Agents and Multi-Agent Systems(22): 4-30.

[3] Arrow, K. J. 1963. Social Choice and Individual Values. New York: Wiley, 2d edition.

[4] Dietrich, F. 2007. A generalised model of judgment aggregation. Social Choice and Welfare 28(4):529-565.

[5] Dietrich, F., \& Mongin, P. 2010. The premise-based approach to judgment aggregation. Journal of Economic Theory 145(2):562-582.

[6] Ehlers, L. \& Storcken, T. 2008. Arrow's possibility theorem for one dimensional single-peaked preferences. Games and Economic Behavior, 64(2), 533-547.

[7] Gaertner. W. 2009. A Primer in Social Choice Theory. New York: Oxford University Press.

[8] Grossi, D. 2008. Correspondences in the Theory of Aggregation. Lecture Notes in Computer Science, 2010, Volume 6006, Logic and the Foundations of Game and Decision Theory - LOFT 8, Pages 34-60.

[9] List, C. \& Pettit, P. 2002. Aggregating Sets of Judgments: An Impossibility Result. Economics and Philosophy 18: 89-110.

[10]List, C. 2003. A Possibility Theorem on Aggregation over Multiple Interconnected Propositions. Mathematical Social Sciences 45(1): 1-13. 